\title{
Falsifying Baryogenesis Mechanisms through Observation of Lepton Number and Flavor Violation
}

\author{
Frank F. Deppisch ${ }^{* \dagger}$ \\ University College London, United Kingdom \\ E-mail: f.deppisch@ucl.ac.uk
}

\begin{abstract}
The presence of $(B-L)$ violating interactions is a necessary ingredient in many models trying to explain the observed baryon asymmetry of the universe, but if they are found to be strong they herald the occurrence of a temperature range where any pre-existing $(B-L)$ asymmetry is washed out. We demonstrate in a model-independent approach that the observation of lepton number violating processes can rule out or strongly disfavor certain mechanisms of baryogenesis, including leptogenesis scenarios. We will especially focus on non-standard mechanisms of neutrinoless double beta decay as well as potential lepton number violating processes at the LHC. If such processes are observed associated to a certain energy scale $\Lambda_{\mathrm{LNV}}$, baryogenesis mechanisms acting at higher scales will be generally disfavored. We will further describe how the argument can be strengthened by using information from lepton flavor violating processes such as $\mu \rightarrow e \gamma$. If additionally observed, it would indicate the presence of interactions that equilibrate different lepton flavors, closing the loophole of asymmetries being stored in different lepton flavors. We will outline in detail how baryogenesis mechanisms are affected by certain observations.
\end{abstract}

38th International Conference on High Energy Physics

3-10 August 2016

Chicago, USA

\footnotetext{
* Speaker.

$\dagger$ The speaker would like to thank the organizers of ICHEP 2016 for the opportunity to present this work and to contribute to the proceedings. He also thanks L. Graf, J. Harz, M. Hirsch, Wei-Chih Huang and Heinrich Päs for the collaboration on which this work is based.
} 
The observed matter-antimatter asymmetry of the universe cannot be understood with Standard Model physics. A large number of possible mechanisms to generate the observed asymmetry have been proposed. An interesting scenario is leptogenesis [1]. In its original formulation, the out-of-equilibrium and $C P$ violating decay of the heavy Majorana neutrinos in the type-I seesaw mechanism create a lepton asymmetry which is then converted into a baryon asymmetry through $(B+L)$ violating electroweak sphaleron processes [2].

The presence of lepton number violation (LNV) is a crucial ingredient in leptogenesis. Vice versa, the observation of LNV would have important consequences on the viability of baryogenesis models in general; specifically, it is possible to falsify a large class of high-scale baryogenesis scenarios if LNV was observed at the LHC [3]. For example if a resonant LNV process with the signature $p p \rightarrow l^{ \pm} l^{ \pm} j j$ is observed, its LHC cross section $\sigma_{\mathrm{LHC}}$ is related to the induced lepton asymmetry washout rate $\Gamma_{W} / H$ (relative to the expansion of the universe) [3],

$$
\log _{10}\left(\Gamma_{W} / H\right) \gtrsim 6.9+0.6\left(M_{X} / \mathrm{TeV}-1\right)+\log _{10}\left(\sigma_{\mathrm{LHC}} / \mathrm{fb}\right) .
$$

Here $M_{X}$ is the mass of the hypothetically observed resonance. If $\Gamma_{W} / H \gg 1$, the dilution of a primordial net lepton number density, understood to be produced in a baryogenesis mechanism at a higher scale, is highly effective and the lepton asymmetry would be washed out before sphaleron processes take effect. This is also illustrated in Fig. 1 showing $\Gamma_{W} / H$ as a function of the observed LNV resonance mass $M_{X}$ and the process cross section $\sigma_{L H C}$. Observation of LNV at the LHC would therefore strongly constrain baryogenesis scenarios above the scale $M_{X}$.

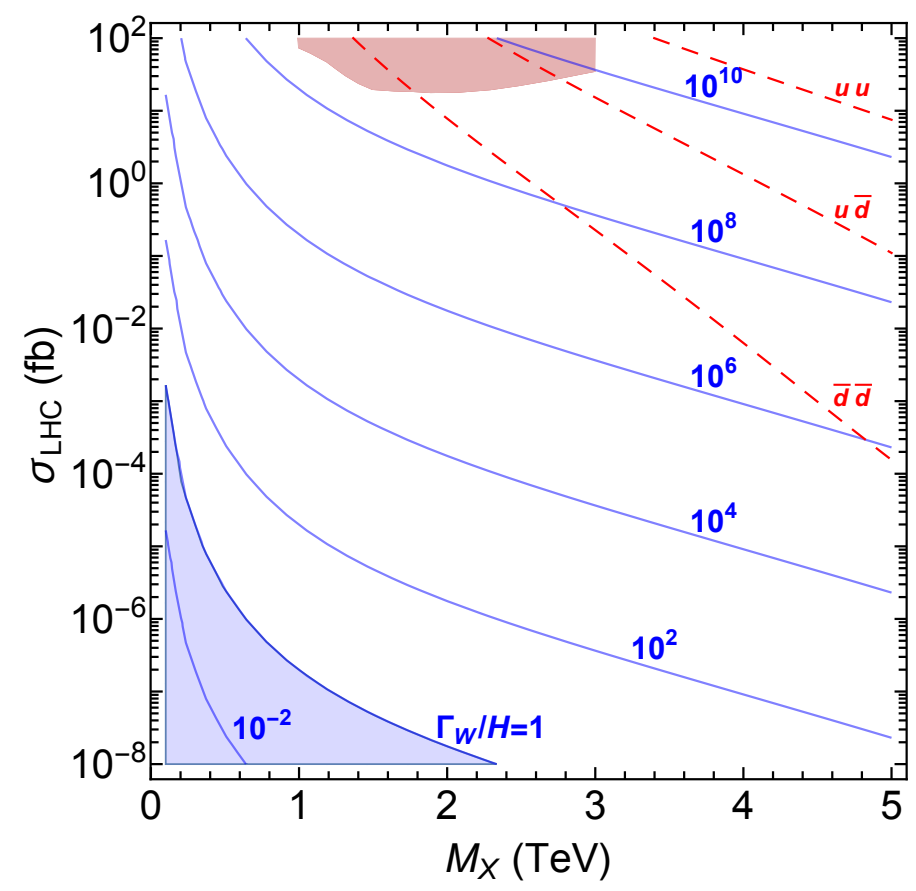

Figure 1: Lepton number washout rate as function of the corresponding LHC cross section and resonance mass (solid blue contours). The dashed red curves give typical cross sections of resonances produced through the quark channels $u u, u \bar{d}$ and $\bar{d} \bar{d}$ with gauge coupling strength for comparison. Taken from [3]. 


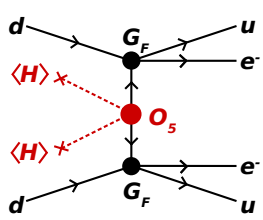

(a)

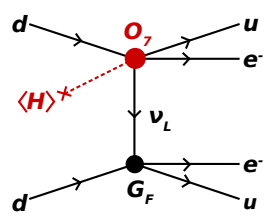

(b)

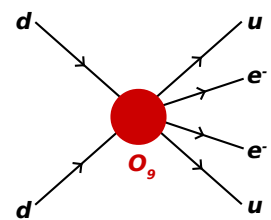

(c)

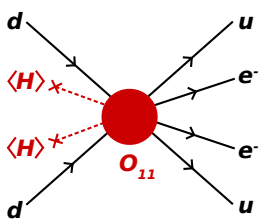

(d)

Figure 2: Contributions to $0 v \beta \beta$ decay generated by effective dimension-5 (a), dimension-7 (b), dimension9 (c) and dimension-11 (d) operators.

A similar argument can be applied to non-standard mechanisms mediating $0 v \beta \beta$ decay and low energy LFV processes [4]: if observed, the corresponding processes would be in equilibrium in certain temperature ranges. The low energy $0 v \beta \beta$ decay can in general be triggered by effect LNV operators of dimension 5 (the Weinberg operator leading to the standard light neutrino exchange), dimension 7 (generically leading to long-range contributions again mediated by light neutrinos) and higher odd-dimensional operators (generically leading to short range operators). Typical contributions, up to dimension 11, are graphically displayed in Fig. 2. Current $0 v \beta \beta$ decay searches probing half lives $T_{1 / 2}^{0 v \beta \beta} \gtrsim 10^{26} \mathrm{yr}$ are roughly sensitive to the scales $\Lambda_{5} \approx 10^{14} \mathrm{GeV}$ (seesaw scale), $\Lambda_{7} \approx 10^{4} \mathrm{GeV}$ and $\Lambda_{9,11} \approx 10^{3} \mathrm{GeV}$, of these operators. Similarly, the presence of any of these operators will also lead to a washout of lepton number in the early universe. As in the case of LNV at the LHC, the $0 v \beta \beta$ decay rate can be related to the washout rate. This is shown in Fig. 3

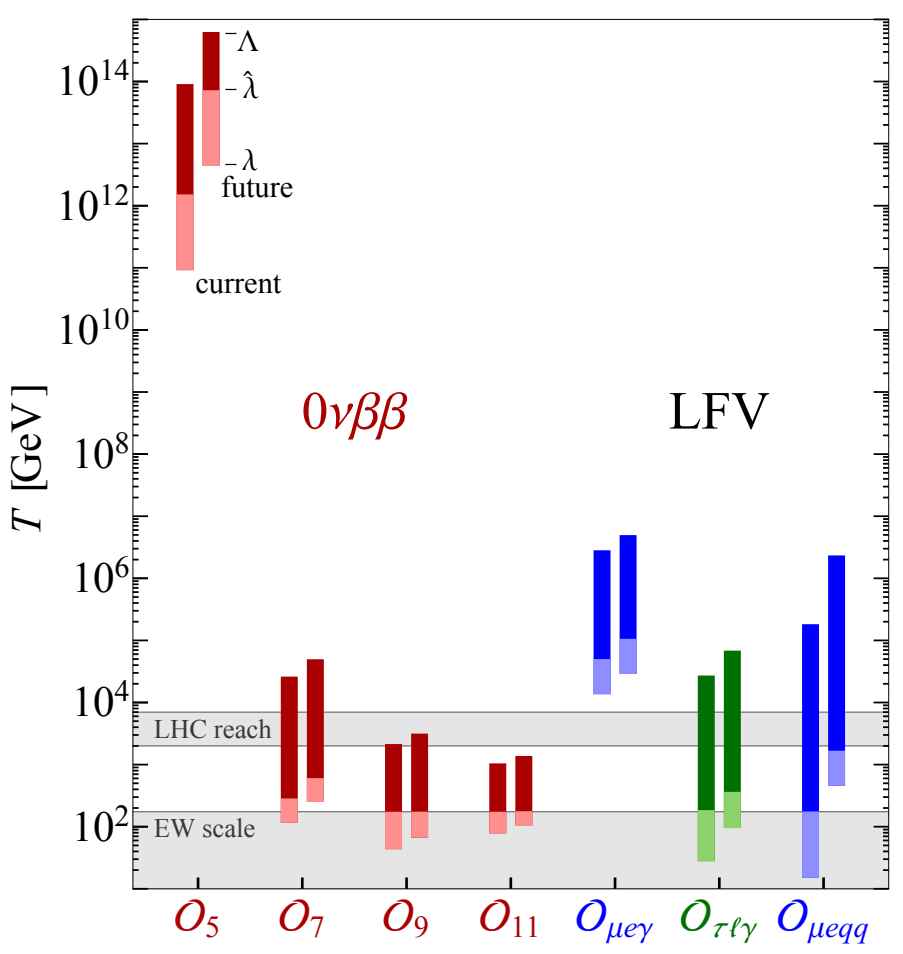

Figure 3: Temperature intervals where the given LNV and LFV operators are in equilibrium assuming that the corresponding process is observed at the current or future experimental sensitivity. Taken from [4]. 
where the coloured bars denote the efficient equilibration temperatures assuming the relevant observable is seen at the current (left bar) or expected future $\left(T_{1 / 2}^{0 v \beta \beta} \approx 10^{27} \mathrm{yr}\right.$, right bar) sensitivity. In the case of the 7,9,11-dimensional effective operators $\mathscr{O}_{7,9,11}$ mediating $0 v \beta \beta$ decay, an electron lepton asymmetry present at energies higher than the electroweak scale would be washed out.

Observation of LFV via 6-dimensional lepton flavor violating (LFV) operators at compatible scales would allow to extend the argument to other flavours than the electron. The most stringent limits on LFV are currently set on 6-dimensional $\Delta L=0$ operators $\mathscr{O}_{\mu e \gamma}, \mathscr{O}_{\tau l \gamma}$ and $\mathscr{O}_{\mu e q q}$ leading to the following decays and current experimental limits: $\mathrm{Br}_{\mu \rightarrow e \gamma}<5.7 \times 10^{-13}$ [5], $\mathrm{Br}_{\tau \rightarrow \ell \gamma} \lesssim 4.0 \times$ $10^{-8}(\ell=e, \mu)[6], \mathrm{R}_{\mu \rightarrow e}^{\mathrm{Au}}<7.0 \times 10^{-13}$ [6]. The expected sensitivities of ongoing and planned experiments are $\mathrm{Br}_{\mu \rightarrow e \gamma} \approx 6.0 \times 10^{-14}$ [7], $\mathrm{Br}_{\tau \rightarrow \ell \gamma} \approx 1.0 \times 10^{-9}$ [8] and $\mathrm{R}_{\mu \rightarrow e}^{\mathrm{Al}} \approx 2.7 \times 10^{-17}$ [9]. In a similar analysis to the above discussed LNV operators, one can relate the rare LFV process rate with the corresponding flavor equilibration temperature range. In Fig. 3, the flavor equilibration ranges are shown assuming current and future LFV process sensitivities, alongside the electron lepton asymmetry washout of the $0 v \beta \beta$ LNV operators. If LNV and LFV is present at similar scales, the washout of the electron lepton number will thus be transmitted to muons or taus.

Searches for LNV in $0 v \beta \beta$ decay, at the LHC and in other contexts (for example LNV meson decays) are thus powerful tools to narrowing down models of baryogenesis. If $0 v \beta \beta$ decay was observed via a non-standard mechanism, it would point us to low-scale baryogenesis as well as a probable discovery of LNV at the LHC. If however, high-scale baryogenesis is realised in nature, no LNV is expected to be discovered at the LHC. If $0 v \beta \beta$ decay was observed, its underlying mechanism is then likely to be the standard mass mechanism via the Weinberg operator and it would point us to a high-scale origin of neutrino masses. Loop holes in this reasoning exist and we would like to refer the reader to $[3,4]$ for a more detailed discussion.

\section{References}

[1] M. Fukugita and T. Yanagida, Phys. Lett. B174, 45 (1986).

[2] V. A. Kuzmin, V. A. Rubakov, and M. E. Shaposhnikov, Phys. Lett. B155, 36 (1985).

[3] F. F. Deppisch, J. Harz, and M. Hirsch, Phys.Rev.Lett. 112, 221601 (2014), 1312.4447; F. F. Deppisch and J. Harz, (2014), 1408.5351.

[4] F. F. Deppisch, J. Harz, M. Hirsch, W.-C. Huang, and H. Päs, Phys. Rev. D92, 036005 (2015), 1503.04825 .

[5] J. Adam et al. [MEG Collaboration], Phys. Rev. Lett. 110 (2013) 201801.

[6] K. A. Olive et al. [Particle Data Group Collaboration], Chin. Phys. C 38 (2014) 090001.

[7] A. M. Baldini et al., arXiv:1301.7225 [physics.ins-det].

[8] T. Aushev et al., arXiv:1002.5012 [hep-ex].

[9] R. Akhmetshin et al. [COMET Collaboration], http://comet.kek.jp/Documents_files/PAC-TDR-2014/PAC-Review-20141110.pdf.

[10] M. Doi, T. Kotani, H. Nishiura and E. Takasugi, Prog. Theor. Phys. 69 (1983) 602. 